\title{
Fatty acid-binding protein 5 predicts poor prognosis in patients with uveal melanoma
}

\author{
YUE XU ${ }^{1,2^{*}}$, WEN-HAO XU ${ }^{3,4^{*}}$, XIAO-LONG YANG ${ }^{1,2}$, HAI-LIANG ZHANG $^{3,4}$ and XIAO-FENG ZHANG ${ }^{1,2}$ \\ ${ }^{1}$ Department of Ophthalmology, The First Affiliated Hospital of Soochow University; ${ }^{2}$ Department of Ophthalmology, \\ Soochow University Medical College, Soochow University, Suzhou, Jiangsu 215000; \\ ${ }^{3}$ Department of Urology, Fudan University Shanghai Cancer Center; ${ }^{4}$ Department of Oncology, \\ Shanghai Medical College, Fudan University, Shanghai 20032, P.R. China
}

Received August 28, 2019; Accepted November 29, 2019

DOI: $10.3892 / \mathrm{ol} .2020 .11301$

\begin{abstract}
Fatty acid-binding protein 5 (FABP5), which participates in mediating the biological properties of tumor cells, has been recognized in several neoplasms. The present study aims to investigate $F A B P 5$ transcriptional expression profiles, reveal its underlying biological interaction networks and define its prognostic value in uveal melanoma (UVM). A total of 80 patients with UVM and their RNA-sequence data, available from The Cancer Genome Atlas (TCGA) database, was analyzed. A differential transcriptional expression profile was obtained from TCGA and the Oncomine databases. The survival benefits were analyzed using the Kaplan-Meier method and log-rank test. The correlation between FABP5 expression and immune infiltration level was analyzed using the Tumor Immune Estimation Resource database. Functional enrichment analyses using Gene Ontology, Kyoto Encyclopedia of Genes and Genomes, and signaling hallmarks were utilized to describe the biological process, molecular functions, cellular component and significantly involved pathways. The elevated transcriptional expression of FABP5 was significantly associated with shorter overall survival (OS) and worse progression-free survival (PFS) times in patients with UVM $(\mathrm{P}<0.001)$. Moreover, FABP5 expression was
\end{abstract}

Correspondence to: Dr Xiao-Feng Zhang, Department of Ophthalmology, The First Affiliated Hospital of Soochow University, 188 Shizi Street, Suzhou, Jiangsu 215000, P.R. China E-mail: zhangxiaofeng@suda.edu.cn

Abbreviations: UVM, uveal melanoma; FABP5, fatty acid-binding protein 5; TCGA, The Cancer Genome Atlas; PFS, progression-free survival; OS, overall survival; GO, Gene Ontology; BP, biological processes; CC, cellular components; MF, molecular function; KEGG, Kyoto Encyclopedia of Genes and Genomes; PPI, protein-protein interaction; GSEA, Gene Set Enrichment Analysis

*Contributed equally

Key words: uveal melanoma, fatty acid-binding protein 5, biological markers, prognosis, mRNA significantly and positively correlated with tumor purity and $\mathrm{CD}^{+} \mathrm{T}$ cells and was negatively correlated with the infiltrating levels of $\mathrm{CD}^{+} \mathrm{T}$ cells and neutrophils. Gene Set Enrichment Analysis was performed to obtain 100 significantly associated genes of FABP5 and FABP5 was found to be critical in several hallmark pathways, including allograft rejection, complement, interleukin-6/Janus kinase-STAT3 signaling, interferon $\gamma$ response, inflammatory response and tumor necrosis factor $\alpha$ signaling via $\mathrm{NF} \kappa \mathrm{B}$. The present study is the first to demonstrate that FABP5 expression was positively associated with progression-associated clinicopathological factors and poor prognosis in UVM, which suggests its likely function as an oncogene and prognostic marker in patients with UVM.

\section{Introduction}

Uveal melanoma (UVM) is the cause of $~ 85 \%$ of all ocular melanomas and is the most common primary intraocular malignancy in adults (1). The average annual incidence of UVM in the US is 5.1 per million between 1973 and 2012 (95\% CI, 4.2-6.1) (2). Approximately $50 \%$ of patients with UVM develop metastatic disease (3), with the liver being the most common initial site of metastasis. Patients with metastatic disease are rarely candidates for curative surgery and generally have a poor prognosis; death often occurs within a few months of the development of metastases $(4,5)$. Although the incidence rate of UVM is known to be influenced by a number of parameters, including demographic, geographic and, to a lesser extent, hereditary factors, little is known about the underlying mechanisms responsible for its initiation, progression or biological heterogeneity. Consequently, there is an urgent need to increase the understanding of the molecular and cellular biology of UVM, which will aid in the development not only of novel prognostic biomarkers but also of individualized treatment regimens.

Fatty acid-binding proteins (FABPs) are a protein family (6) that bind to hydrophobic lipids, including various retinoids and long-chain fatty acids and are involved in lipid metabolism (7) by affecting lipid transport, storage, membrane incorporation and transcriptional regulation $(8,9)$. The $F A B P 5$ isoform is an intracellular lipid-binding protein that is highly expressed in macrophages and adipocytes (10). FABP5 is transcriptionally 
regulated by a number of cytokines and signaling pathways, including the phosphoinositide 3-kinase (PI3K)/AKT pathway and the transcription factors peroxisome proliferator-activated receptor (PPAR) $\beta / \delta$ and nuclear factor $\kappa$ light chain enhancer of activated B cells (NFKB) (11-13). Notably, FABP5 was overexpressed in several tumor types and its expression level was associated with the growth and metastasis of several cancer types, including prostate cancer, intrahepatic cholangiocarcinoma, colorectal cancer and cervical cancer $(7,8,12,14,15)$.

An understanding of the regulation and function of FABP5 in normal organ development and disease progression may identify novel targets for UVM treatment. To investigate the transcriptional expression of FABP5 and define its prognostic value in patients with UVM, the present study focused on analyzing the gene expression profiles, revealing the underlying biological interaction networks and assessing their prognostic value. It is postulated that the potential oncogenic activity of FABP 5 correlates with poor prognosis and might reveal its potential therapeutic targets and the molecular pathogenesis of UVM.

\section{Materials and methods}

Patients and transcriptional expression profile. RNA-sequence data, from The Cancer Genome Atlas (TCGA) database (16), including 80 patients with UVM were downloaded and analyzed. The gene expression profile was detected experimentally using the Illumina HiSeq-2000 RNA Sequencing platform by the University of North Carolina TCGA genome characterization center. The X-tile software (version 3.6.1) was used to determine the cut-off value of mRNA expression of FABP5 by assessing the biological relationships between FABP 5 mRNA expression levels and the outcome of UVM patients (17). The differential transcriptional expression levels of $F A B P 5$ between patients with metastatic and non-metastatic UVM, from the GSE22138 dataset, was acquired from Oncomine database (18).

Oncomine database. The transcriptional expression profiles of FABP5 in patients with UVM were publicly available from the Oncomine online database (http://www.oncomine. com), which was used to illustrate the differential expression in patients with metastatic and non-metastatic UVM (19). The expression of $F A B P 5$ profiles from the Oncomine database was obtained based on the following criteria: i) 'Gene: FABP5'; ii) 'Cancer Type: Uveal Melanoma'; iii) 'Data Type: mRNA'; iv) Threshold Setting Condition ( $\mathrm{P}<0.0001$; fold change, $>2$; and gene rank, top 10\%); and v) Group by 'Metastatic Event Status'.

Statistical analysis. The phenotype and expression profiles of FABP5 in 80 patients with UVM from TCGA and Oncomine databases were analyzed and presented. The transcriptional expression levels of FABP5 in UVM and their association with clinicopathological parameters (age of the patients, tumor histology and individual cancer stages), obtained from TCGA, were analyzed and compared among different groups visually using a $\chi^{2}$ test. The differential transcriptional expression levels of FABP5 between patients with metastatic and non-metastatic UVM, from the GSE22138 dataset acquired from Oncomine database (18), was analyzed using Student's t-test. Survival comparison between distinct mRNA expression levels groups of $F A B P 5$ was analyzed in patients with UVM from TCGA database. Overall survival (OS), which was evaluated from the date of first therapy to the date of death or last follow-up, was the primary end point. The secondary end point was progression-free survival (PFS), which was the duration between the onset of curative treatment and the date of progression or second-line treatment or death, whichever occurred first. The follow-up duration was evaluated using the Kaplan-Meier method with log-rank test and 95\% CI of the separate curves. Partial Spearman's correlation and statistical significance were calculated for the correlation analysis between FABP5 expression levels and immune infiltration levels. The hypothetical tests were bilateral and $\mathrm{P}<0.05$ was considered to indicate a statistically significant difference. The receiver operating characteristic curve (ROC) was constructed by predicting the probability of a diagnosis being of high or low integrated score of significant hub gene expression. The area under curve (AUC) analysis was used to assess the diagnostic ability.

Tumor immune estimation resource (TIMER) database analysis. The correlation between FABP5 expression and the abundance of immune infiltrates in UVM was analyzed using TIMER (cistrome.shinyapps.io/timer/), which is an integrated resource for the scientific analysis of immune infiltrates across multiple cancer types (20). TIMER applies a previously published deconvolution statistical method to infer several tumor-infiltrating immune cells from gene expression profiles (21). The TIMER database includes 10,897 samples across 32 cancer types from TCGA, enabling the evaluation of the abundance of immune infiltrates. The correlation between $F A B P 5$ expression and the various immune infiltrates, including $\mathrm{CD}^{+} \mathrm{T}$ cells, $\mathrm{CD} 4^{+} \mathrm{T}$ cells and neutrophils, were analyzed via gene modules. The gene expression levels against tumor purity are displayed on the left-most panel (22). Tumor purity is the proportion of cancer cells in the admixture. Genes highly expressed in the microenvironment are expected to have negative associations with tumor purity, whereas genes highly expressed in the tumor cells are expected to have positive associations with tumor purity.

Protein-protein interaction (PPI) network construction and module analysis. PPIs are physical contacts of high specificity that are established between proteins, as a result of biochemical events steered by electrostatic forces. The PPI network is essential in understanding cell physiology in normal and disease states and for drug development. The Search Tool for the Retrieval of Interacting Genes (http://string-db.org; version 10.0) online database was used to predict the PPI network of co-regulated hub genes and for analyzing the functional interactions between proteins (23). An interaction with a combined score of $>0.4$ was regarded as statistically significant.

In order to detect the potential functions, the Gene Ontology (GO) biological process (BP), cellular component (CC), molecular function (MF) and the Kyoto Encyclopedia of Genes and Genomes (KEGG) pathway analyses of hub genes in this module were analyzed using the Database for Annotation, Visualization and Integrated Discovery (http://david.ncifcrf.gov; 
A

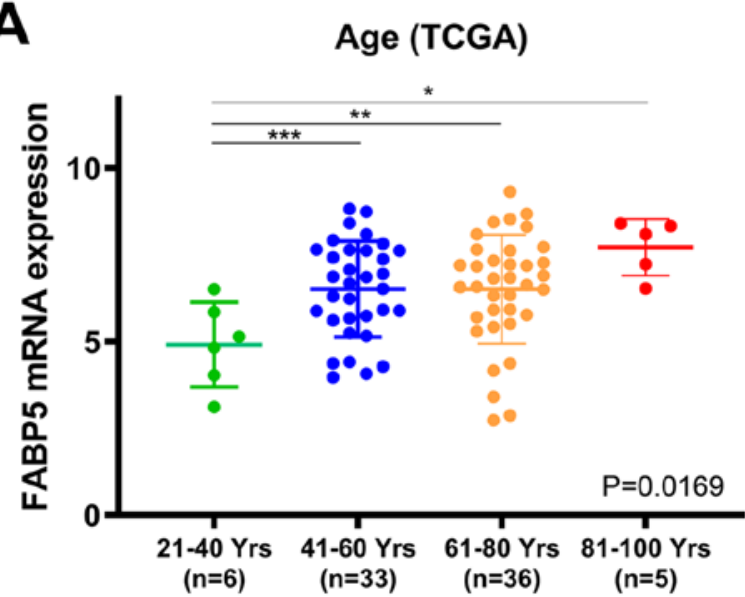

C

Individual cancer stages (TCGA)

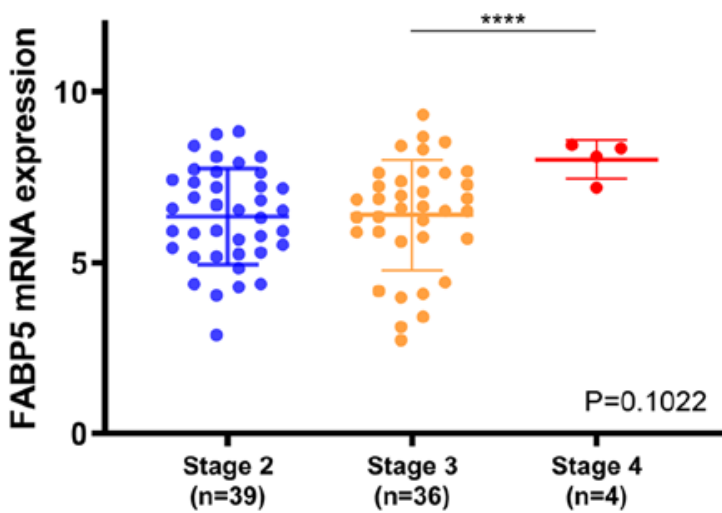

B

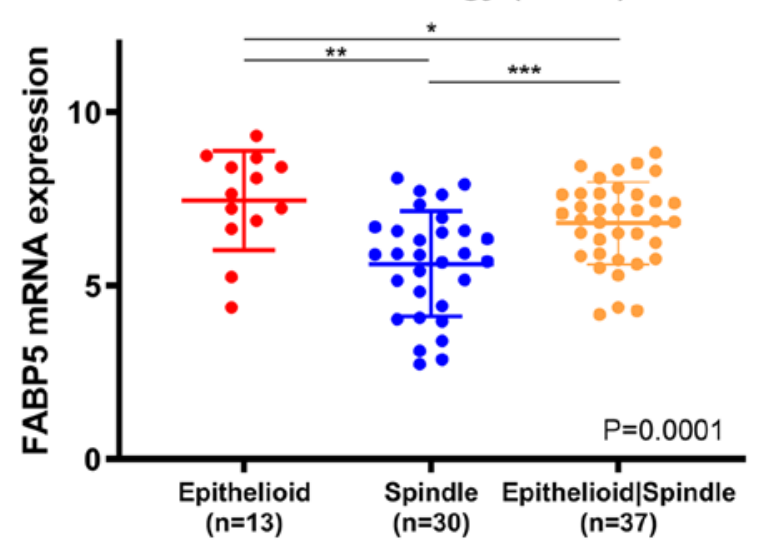

D

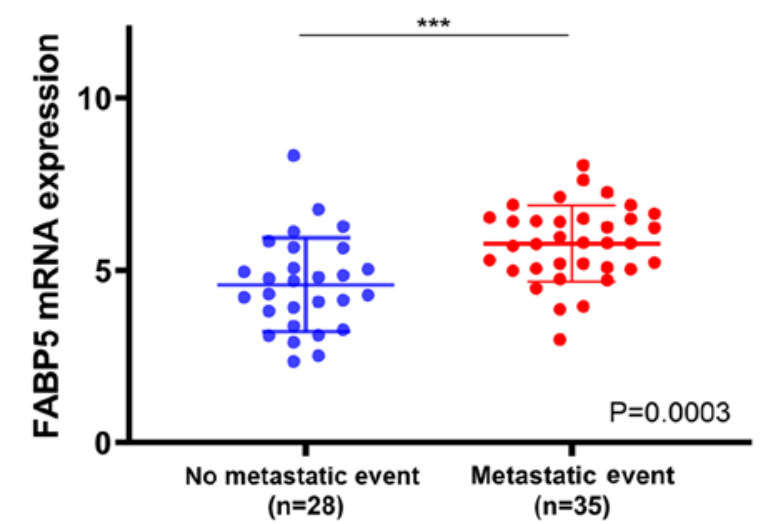

Figure 1. Transcriptional expression of $F A B P 5$ was significantly associated with clinicopathological parameters in patients with UVM. (A and B) Transcriptional expression of FABP5 in UVM (data from TCGA) was significantly associated with the age of the patient ( $\mathrm{P}=0.0169)$ and tumor histology ( $\mathrm{P}=0.0001)$. (C) Transcriptional expression of FABP5 in UVM (data from TCGA) was not significantly associated with individual cancer stages (P=0.1022). However, the highest mRNA expression of $F A B P 5$ was found in stage 4, which was significantly higher compared with that in stage 3 . (D) Transcriptional expression of FABP5 in UVM (data from GSE22138) was significantly higher in the metastatic group $(\mathrm{P}=0.0003) .{ }^{*} \mathrm{P}<0.05,{ }^{* *} \mathrm{P}<0.01,{ }^{* * *} \mathrm{P}<0.001,{ }^{* * * *} \mathrm{P}<0.0001 . F^{2} \mathrm{BP}$, fatty acid-binding protein 5; UVM, uveal melanoma; TCGA, The Cancer Genome Atlas.

version 6.8) online tool (24) and subsequently visualized using a bubble chart. $\mathrm{P}<0.05$ was considered to indicate a statistically significant difference.

Hierarchical partitioning was performed on the transcriptional expression profiles of eleven hub genes using a heat map. The color gradients illustrate high (blue) or low (yellow) expression levels.

Data processing of gene set enrichment analysis (GSEA). GSEA was used to determine whether the differential expression of FABP5 was associated with a particular biological process or molecular function. TCGA database was implemented with the GSEA method using the Category version 3.0 package (25). Student's-t-test was performed for every separate analysis in consistent pathways and the mean of the differentially expressed genes was calculated. A total of 1,000 permutation tests were used to identify pathways with significant changes. The adjusted P-values (adj. P) with Benjamini and Hochberg (BH) false discovery rate (FDR) method by default were utilized to correct the occurrence of false positive results (25). The significantly associated genes were defined with an adj. $\mathrm{P}<0.01$ and FDR $<0.25$. Statistical analysis and graphical plotting were conducted using the $\mathrm{R}$ software (version 3.3.2).

\section{Results}

Transcriptional expression of FABP5 in UVM based on clinicopathological parameters. As illustrated in Fig. 1, the transcriptional expression profiles of FABP5 from the RNA-sequence data from TCGA database and the GSE22138 dataset were analyzed. The transcriptional expression of FABP5 in UVM was significantly associated with the age of the patient $(\mathrm{P}=0.0169)$. The lowest mRNA expression of FABP5 was detected in the 21-40 year age group. The transcriptional expression levels of $F A B P 5$ was found to be higher in the 81-100 $\left({ }^{*} \mathrm{P}<0.05\right)$, the $61-80\left({ }^{* *} \mathrm{P}<0.01\right)$ and the $41-60$ $\left({ }^{* * *} \mathrm{P}<0.001\right)$ age groups compared with that in the $21-40$ age group (Fig. 1A). In addition, the transcriptional expression levels of FABP5 in UVM was significantly associated with 
A

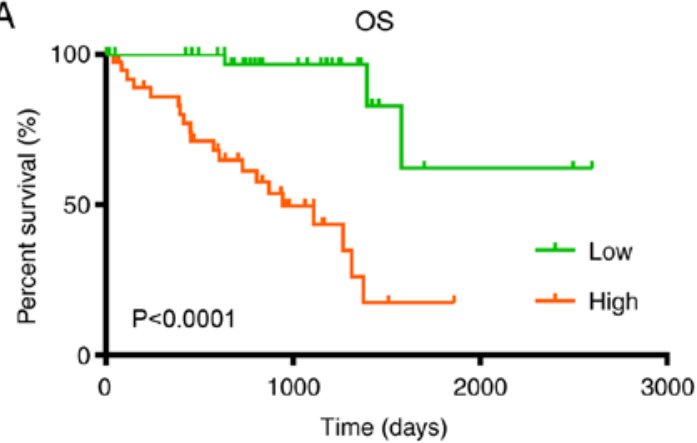

B

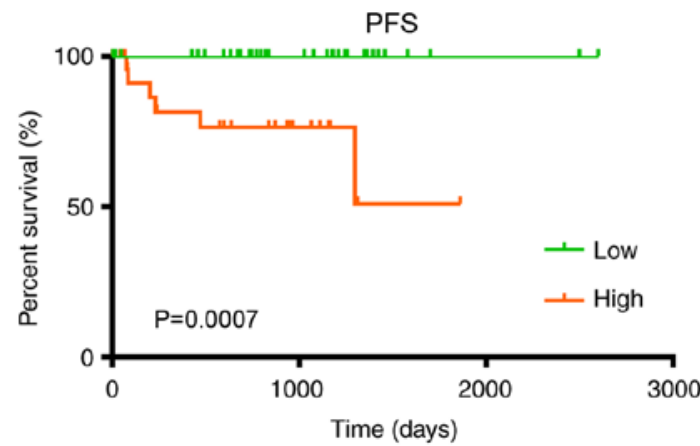

Figure 2. Kaplan-Meier survival analyses of OS and PFS in patients with UVM with differential FABP5 expression groups. Compared with low mRNA expression, high FABP5 expression was significantly associated with poor (A) OS ( $\mathrm{P}<0.0001)$ and (B) PFS ( $\mathrm{P}=0.0007)$ times. FABP5, fatty acid-binding protein 5; UVM, uveal melanoma; OS overall survival; PFS, progression-free survival.

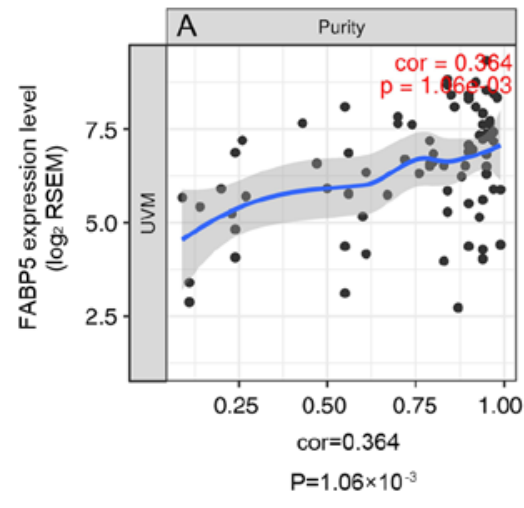

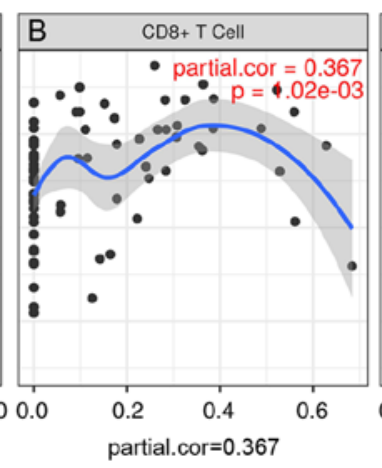

$\mathrm{P}=1.02 \times 10^{-3}$

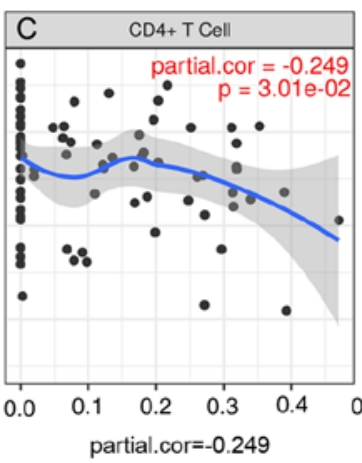

$P=3.01 \times 10^{-2}$

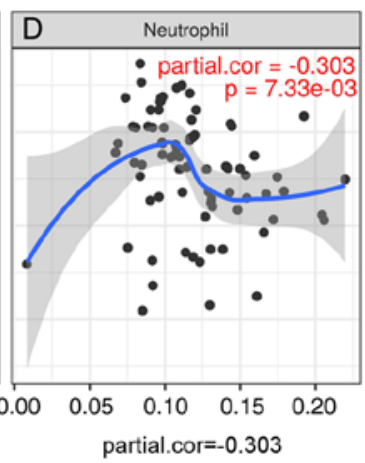

$P=7.33 \times 10^{-3}$

Infiltration level

Figure 3. Correlation between $F A B P 5$ expression and immune infiltration level in UVM. FABP5 expression is significantly and positively correlated with tumor purity (A) and $\mathrm{CD}^{+} \mathrm{T}$ cells (B), whereas significantly and negatively correlated with infiltrating levels of CD4 ${ }^{+} \mathrm{T}$ cells $(\mathrm{C})$ and neutrophils $(\mathrm{D})$ (n=80). FABP5, fatty acid-binding protein 5; UVM, uveal melanoma; partial.cor, purity-corrected partial Spearman's correlation; RSEM, RNA-Seq by expectation-maximization.

tumor histology $(\mathrm{P}=0.0001)$. The highest mRNA expression levels of FABP5 was detected in epithelioid UVM, whereas the lowest level was found in spindle cell UVM. The transcriptional expression levesl of FABP5 was found to be higher in the epithelioid UVM compared with that in the mixed cell UVM $\left({ }^{*} \mathrm{P}<0.05\right)$ and spindle cell UVM groups $\left({ }^{* *} \mathrm{P}<0.01\right)$, and higher in the mixed cell UVM compared with that in the spindle cell UVM $\left({ }^{* * *} \mathrm{P}<0.001\right)$ group (Fig. 1B). The transcriptional expression of $F A B P 5$ in UVM was not significantly associated with individual cancer stages $(\mathrm{P}=0.1022$; Fig. $1 \mathrm{C})$. However, patients who were in more advanced stages tended to express higher mRNA expression levels of FABP5. The highest mRNA expression of FABP5 was found in stage 4, which was significantly higher compared with that in stage 3 $\left({ }^{* * * *} \mathrm{P}<0.0001\right)$. The transcriptional expression of FABP5 in UVM was significantly associated with metastatic event status in GSE22138 ( $\mathrm{P}=0.0003)$. A higher level of FABP5 mRNA expression levels were found in patients with a metastatic event (Fig. 1D). The baseline clinicopathological characteristics, according to FABP5 expression status, are shown in Table SI.

Survival outcomes of the 80 UVM patients from TCGA. The patients have been divided according to FABP5 expression levels, therefore overall survival is associated with expression levels of FABP5 and patients with a high expression levels have a significantly low overall survival time ( $\mathrm{P}<0.001$; Fig. $2 \mathrm{~A})$. In addition, patients with UVM and high FABP5 mRNA levels showed shorter PFS time $(\mathrm{P}=0.0007$; Fig. 2B). Furthermore, the ROC curve was generated to validate the ability of the logistic model to predict prognosis. The AUC index for the integrated model was 0.867 for the $\mathrm{OS}(\mathrm{P}=0.008)$ for patients with UVM who had died (Fig. S1).

Immune infiltration level. Tumor-infiltrating lymphocytes are an independent predictor of cancer sentinel lymph node status and survival rate $(26,27)$. Therefore, the correlation between $F A B P 5$ expression and immune infiltration levels in UVM was investigated using TIMER. The analysis demonstrated that FABP5 expression had significant and positive correlation with tumor purity and $\mathrm{CD}^{+} \mathrm{T}$ cells in UVM and significant negative correlation with infiltrating levels of $\mathrm{CD}^{+} \mathrm{T}$ cells and neutrophils in UVM (Fig. 3).

Functional annotation and predicted signaling pathways. The PPI network of FABP5 was constructed. The network of FABP5 and its co-expressing genes (resistin, ATP citrate lyase, annexin A2, lipase E, Serpin family B member 3, PPAR $\delta$, retinoid $\mathrm{X}$ receptor $\alpha$, transthyretin, granulin precursor and S100 calcium binding protein A7) was visualized (Fig. 4A). The PPI network derived from active interaction sources was 

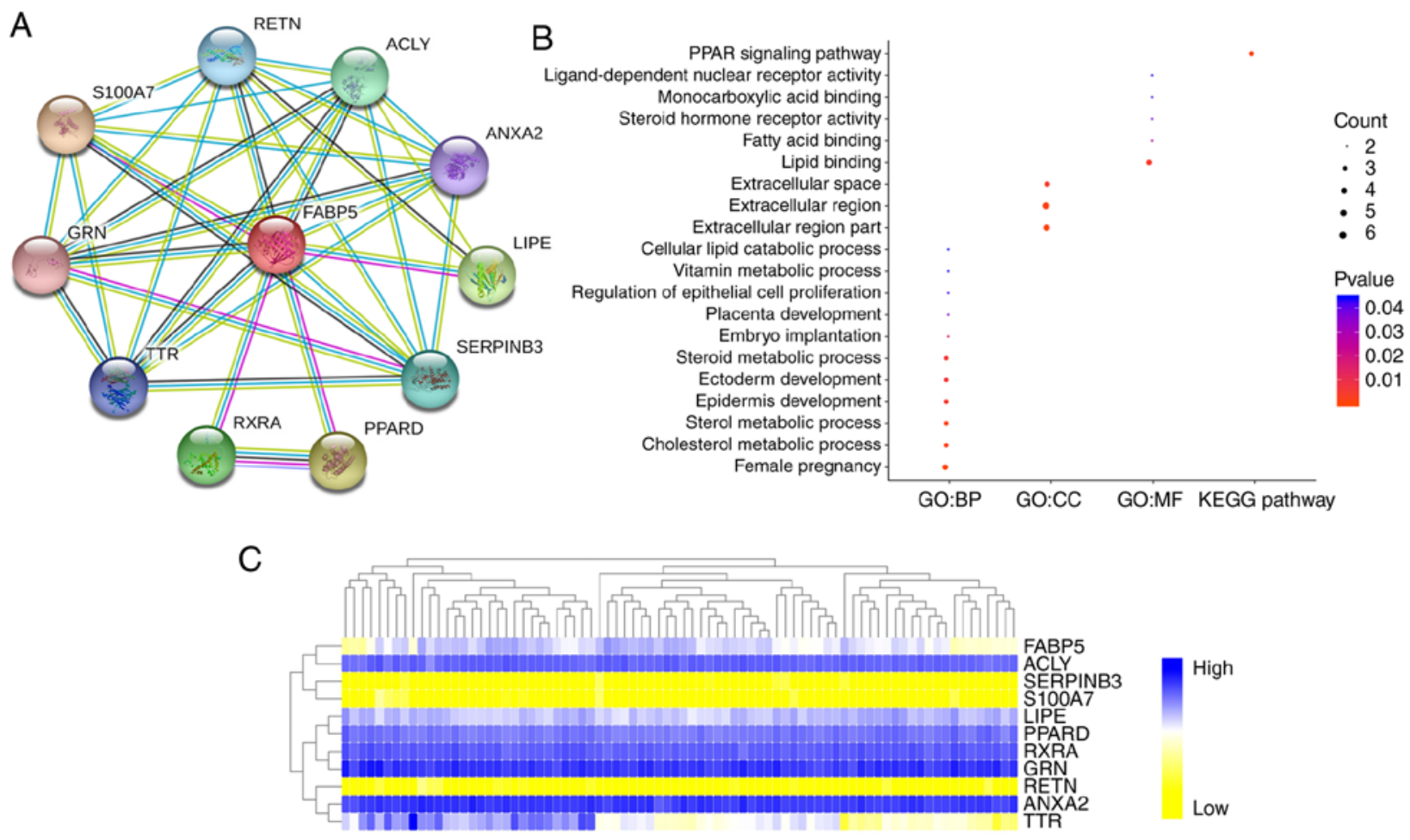

Figure 4. Functional annotations and predicted signaling pathways. (A) The PPI network of $F A B P 5$ was constructed. The network of $F A B P 5$ and its co-expression genes was illustrated visually. (B) Functional and pathway enrichment analyses of a total of 11 genes associated with $F A B P 5$ were performed and visualized using a bubble chart. (C) Hierarchical partitioning of $F A B P 5$ and its co-expressing genes was obtained from DNA microarrays. The expression levels of 11 genes across a number of comparable samples are presented, with high expression marked in blue and low expression marked in yellow. FABP5, fatty acid-binding protein 5; PPI, protein-protein interaction; GO, Gene Ontology; BP, biological processes; MF, molecular functions; CC, cellular components; KEGG, Kyoto Encyclopedia of Genes and Genomes.

illustrated in detail with the required interaction score equal to 0.400 . As illustrated in Fig. 4B, functional and pathway enrichment analyses of a total of 11 associated genes were performed and visualized using a bubble chart. The changes in the BP of significant genes were significantly enriched for 'female pregnancy', 'cholesterol metabolic process', 'sterol metabolic process', 'epidermis development', 'ectoderm development' and 'steroid metabolic process'. The changes in $\mathrm{CC}$ were mostly enriched for the 'extracellular region part', 'extracellular region' and 'extracellular space'. The GO analysis results showed that changes in the MF of significant genes were primarily enriched in 'lipid binding'. The hierarchical partitioning of FABP5 and its co-expressing genes was obtained from 80 UVM patients of TCGA database (Fig. 4C). It represents the levels of expression of 11 genes across 80 comparable UVM patients from TCGA database with high expression marked in blue and low expression marked in yellow.

Significant genes and pathways obtained by GSEA. A total of 100 significantly associated genes were obtained using GSEA, including those with both positive and negative associations. Importantly, GSEA was used to perform the analysis of hallmark pathways that are associated with FABP5. The results suggested the pathways that were significantly associated with $F A B P 5$, included allograft rejection, complement, interleukin-6/Janus kinase-STAT3 signaling, interferon $\gamma$ response, inflammatory response and tumor necrosis factor $\alpha$ signaling via NFkB (Fig. 5A-F). In addition, the transcriptional expres- sion profiles of the 100 significant genes were analyzed using a heat map (Fig. 5G).

\section{Discussion}

Aberrant genetic and epigenetic regulation of key metabolic pathways is known to contribute towards the development and progression of UVM (28). Elevated expression of the protease A disintegrin and metalloproteinase domain 10 and the membrane transporter ATP binding cassette subfamily B member 5 was shown to be associated with rapid metastatic progression and worse prognosis in UVM, suggesting that these proteins may be useful as prognostic factors $(29,30)$. As a major mediator of fatty acid uptake, transport and metabolism, FABP5 may participate in the development and aggressive behavior of cancer $(7,12,31,32)$. Accordingly, FABP5 is known to play an oncogenic role in numerous types of cancer, including prostate carcinogenesis, cervical cancer, renal cell carcinoma and hepatocellular carcinoma $(6,8,11,12)$, however to the best of our knowledge the prognostic implications of FABP5 expression in UVM are currently unknown. In order to address this gap in knowledge; the present study investigated the expression, potential function and prognostic value of $F A B P 5$ in UVM.

$F A B P 5$ is an intracellular carrier of long-chain fatty acids and other bioactive lipids and also modulates their metabolism. In addition to transporting fatty acids within the cytoplasm, FABP5 transfers fatty acids into the nucleus, where they activate transcription factors (33). For example, FABP5 transfers 


\section{A}

Enrichment plot: HALLMARK_ALLOGRAFT_REJECTION

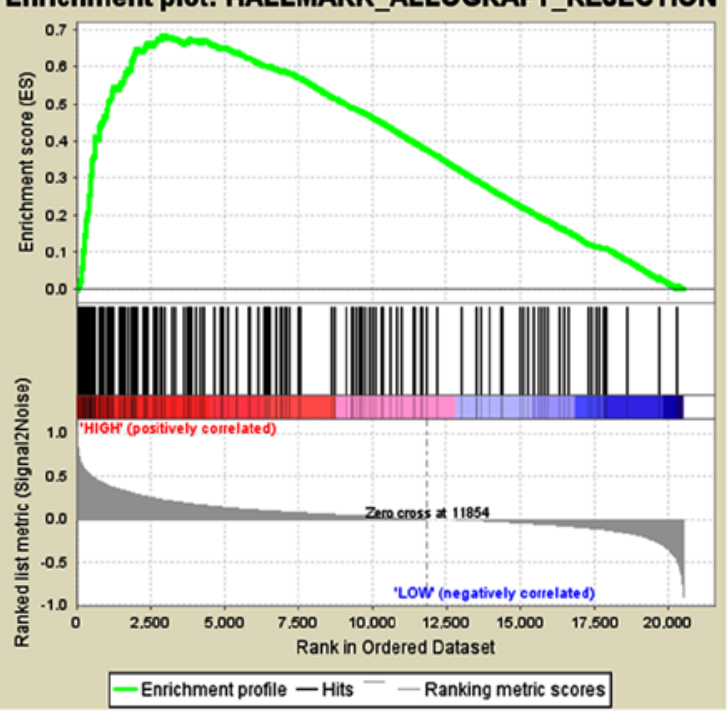

C

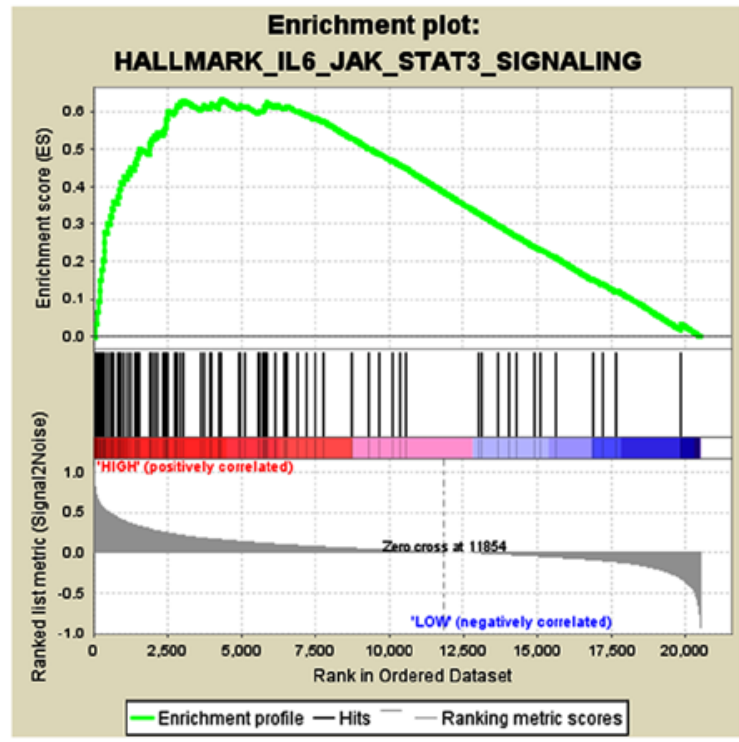

E

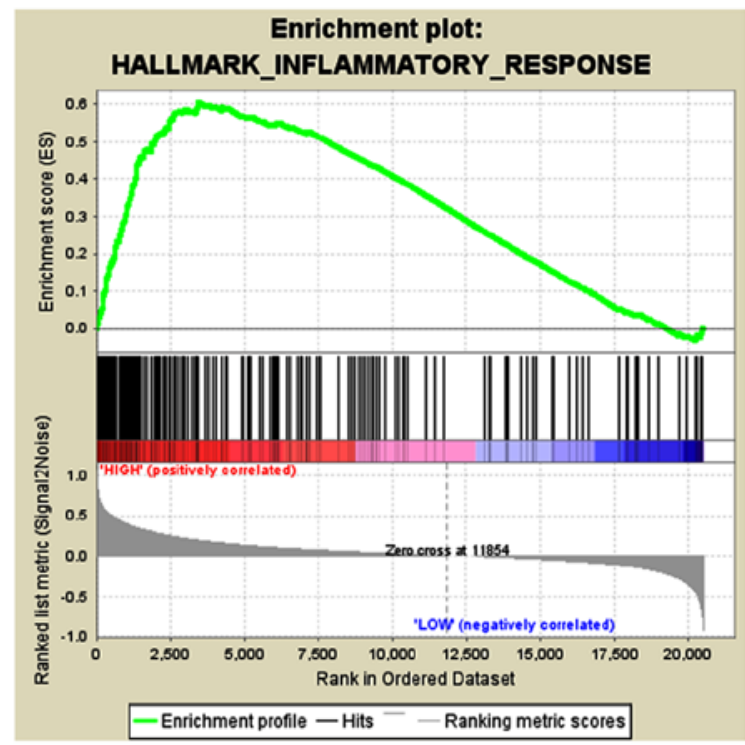

B

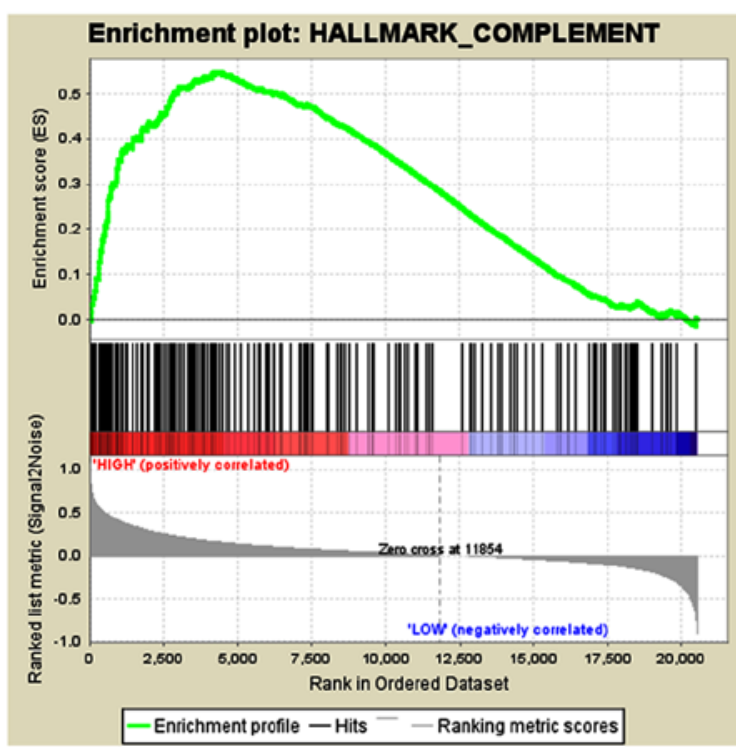

D

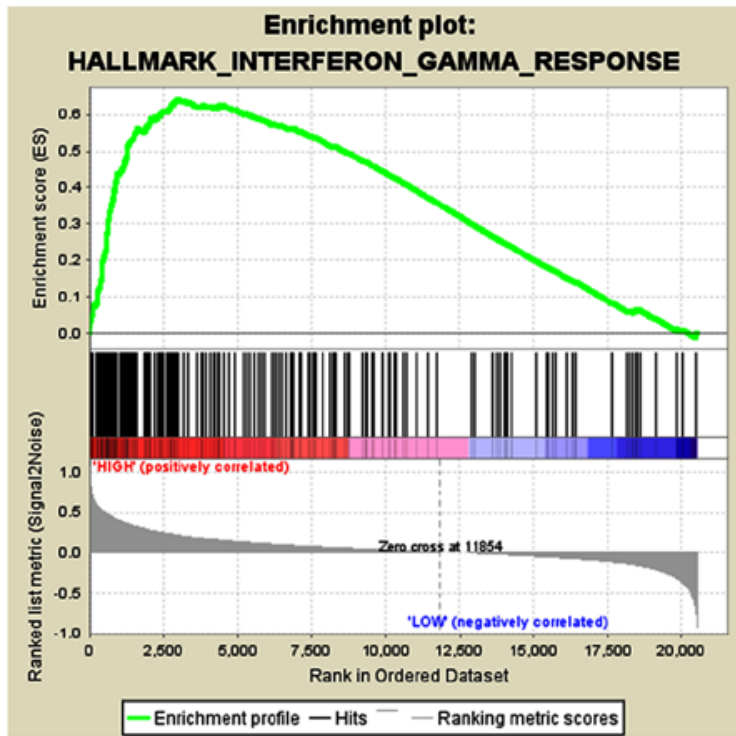

F

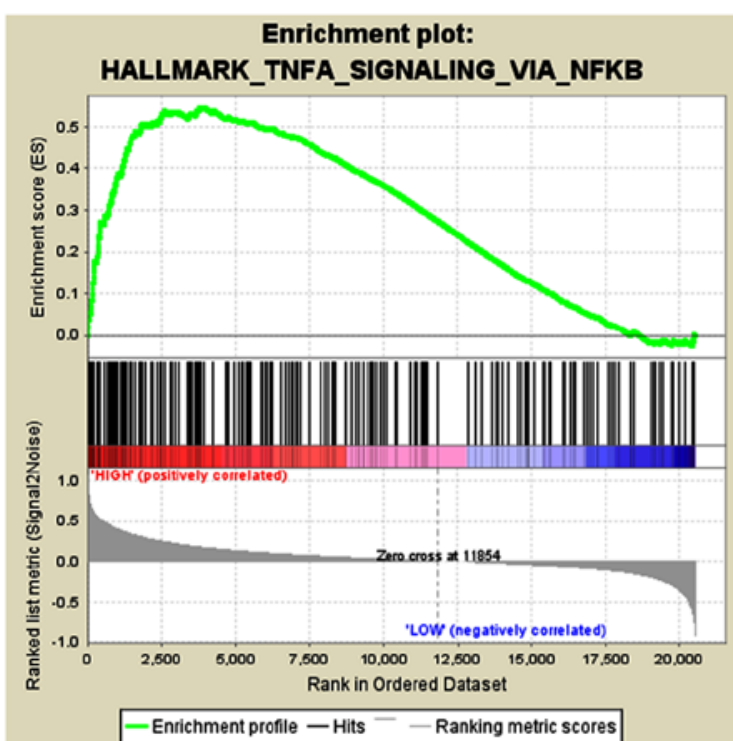

Figure 5. Significantly associated genes and hallmarks pathways in uveal melanoma obtained using GSEA. A total of 100 significant genes were obtained using GSEA with positive and negative associations. (A-F) The most significantly associated pathways included (A) allograft rejection, (B) complement, (C) IL6/JAK-STAT3 signaling, (D) interferon $\gamma$ response, (E) inflammatory response and (F) TNFA signaling via NFkB. GSEA, Gene Set Enrichment Analysis; IL6, interleukin-6; JAK, Janus kinase; TNFA, tumor necrosis factor $\alpha$. 


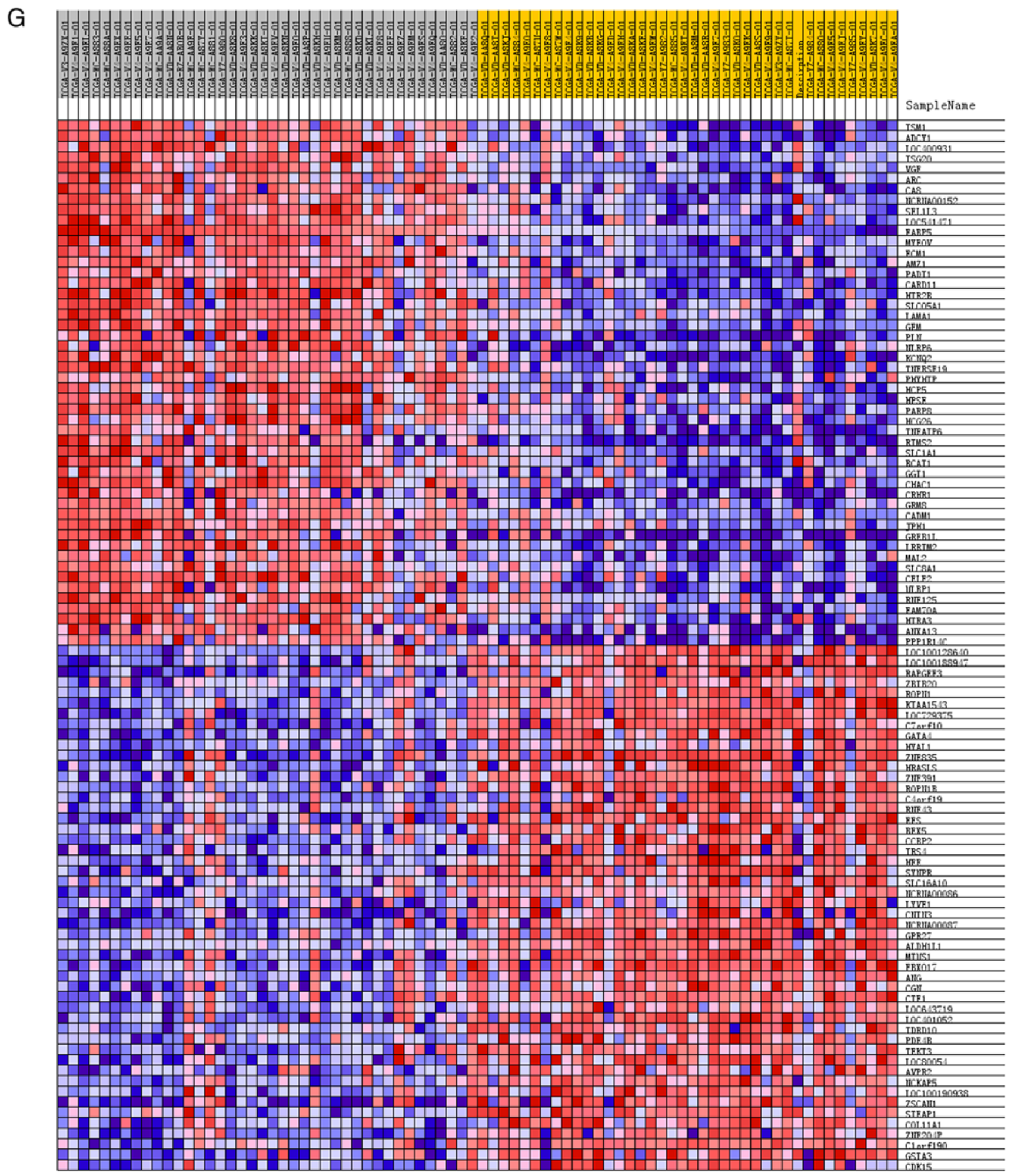

Figure 5. Continued. Significantly associated genes and hallmarks pathways in uveal melanoma obtained using GSEA. A total of 100 significant genes were obtained using GSEA with positive and negative associations. (G) The transcriptional expression profiles of the 100 significant genes are presented as a heat map. The color gradients illustrate high (red) or low (blue) expression levels. GSEA, Gene Set Enrichment Analysis.

retinoic acid to PPAR $\delta$, which contributes to cell survival and proliferation, and FABP5 regulates the induction of prostaglandin E synthase and inflammation via prostaglandin E2 biosynthesis and $\mathrm{NF \kappa B}$ activation (34).

Previous studies have shown that $F A B P 5$ expression was associated with the malignant behavior of multiple types of cancer. Among its oncogenic activities, FABP5 promoted cell migration, proliferation and survival by enhancing the transcriptional activities of nuclear receptor peroxisome proliferator-activated receptor $\beta / \delta$ in human breast cancer cells (MDA-MB-231 cells), human immortalized epidermal cells (HaCaT cells) and human colorectal adenocarcinoma cells (LS-174T cells) (7,35-37). FABP5 expression was associated with primary and metastatic prostate cancer 
and is differentially expressed in primary and metastatic UVM $(38,39)$. Moreover, FABP5 was suggested as a potential therapeutic target for prostate cancer (40). Furthermore, the expression of $F A B P 5$ was elevated in the regional lymph nodes of patients with vulvar carcinoma, suggesting its potential as a prognostic marker gene for this disease (41). FABP5 may contribute to retinoic acid resistance and decrease the anticarcinogenic activities of retinoic acid in breast cancer (42).

At the molecular level, elevated FABP5 expression in fibroblasts was shown to increase PPAR activity, cell proliferation, migration and invasion in breast cancer (43). In human prostate cancer cells (PC-3 cells) and human breast cancer cells (MDA-MB-231 cells), FABP5 contributes to inflammatory cytokine production via protein kinase $\mathrm{C}$ and the $\mathrm{NF} \kappa \mathrm{B}$ signaling pathway in response to elevated levels of reactive oxygen species (10). In addition to PPAR $\beta / \delta$, PI3K/AKT and $\mathrm{NF \kappa B}$ activities are involved in the regulation of $F A B P 5$ activity and expression. FABP5 may increase clear cell renal cell carcinoma cell proliferation, partly via the PI3K/AKT signaling pathway (11). In colorectal cancer, $F A B P 5$ promoted cell growth and metastasis via the PPAR $\beta / \delta$ signaling pathway (15). In addition, FABP5 promoted the expression of secreted proteins associated with tumor malignancy, by activating the NFKB signaling pathway (10).

To the best of our knowledge, the present study is the first to investigate the potential of FABP5 as a prognostic factor of UVM. Although FABP5 has been implicated in the development of numerous types of cancer and other human diseases, including prostate cancer, intrahepatic cholangiocarcinoma, colorectal cancer and cervical cancer $(7,8,12,14,15)$, little is known about its involvement in UVM. The present study demonstrated that the mRNA expression levels of FABP5 was elevated in UVM tissues, which was significantly associated with worse clinicopathological parameters, such as shorter OS and PFS times. Of note, another study demonstrated that patients with spindle cell UVM tumors had longer disease-specific survival compared with those with epithelioid and mixed tumors consisting of epithelioid and spindle cells (44). This association was linked to the expression levels of FABP5 mRNA, with the highest being observed in epithelioid UVM and the lowest in spindle cell UVM. Furthermore, younger patients ( $\leq 20$ years) with UVM at the time of diagnosis were found to have a lower rate of metastasis compared with adults (21-60 years) and older adults (>60 years), which indicated the risk of metastasis gradually increased with increasing age (45-47). In the present study, the transcriptional expression of FABP5 in UVM was significantly associated with the age of the patient suggesting it has prognostic value patients with UVM. Furthermore, two additional major findings from the present study reveal that $F A B P 5$ expression was positively correlated with UVM tumor purity and $\mathrm{CD}^{+} \mathrm{T}$ cells whereas it was negatively correlated with immune cell infiltration; specifically, with the number of $\mathrm{CD}^{+} \mathrm{T}$ cells and neutrophils. These data suggests that FABP5 may play a crucial role in immune cell recruitment to and/or retention within the tumor microenvironment in UVM.

There are several limitations to the present study. Firstly, only FABP5 mRNA expression levels were examined as a potential prognostic biomarker to predict OS and PFS times. Secondly, further validation studies or prospective cohorts should be analyzed to verify the present findings. Finally, despite conducting bioinformatics analysis of functional annotations and enrichment of FABP5-associated pathways, these findings were not verified by exploring the underlying molecular mechanisms of FABP5 signaling. Thus, further studies will be required to understand the association between FABP5 and tumor growth in UVM, as well as in other cancer types.

To the best of our knowledge, the present study is the first to reveal that elevated $F A B P 5$ expression is significantly associated with cancer progression and poor survival in patients with UVM. Thus, FABP5 is a potential marker of UVM, which is easily detected, thereby assisting in the selection of monitoring and treatment strategies. The present study also provides novel directions for further studies, in order to elucidate the molecular pathogenesis of UVM. Such studies, together with randomized clinical trials, will be required to understand the precise underlying mechanisms of action of FABP5 and its clinical application in patients with UVM.

\section{Acknowledgements}

Not applicable.

\section{Funding}

The present study was supported by grants from the National Natural Science Foundation of China (grant nos. 81202004 and 81802525).

\section{Availability of data and material}

The datasets analyzed during the current study are available from the corresponding author on reasonable request.

\section{Authors' contributions}

XFZ designed the research and contributed towards the analyses, interpretation and presentation of data. YX and WHX drafted the manuscript, analyzed the data and interpreted the results. XLY helped to perform the statistical analysis and the literature review. HLZ co-worked on associated data collection, data interpretation and revising the draft. All authors read and approved the final manuscript.

\section{Ethical approval and consent to participate}

Not applicable.

\section{Patient consent for publication}

Not applicable.

\section{Competing interests}

The authors declare that they have no competing interests.

\section{References}

1. Kaliki S and Shields CL: Uveal melanoma: Relatively rare but deadly cancer. Eye (Lond) 31: 241-257, 2017. 
2. Mahendraraj K, Lau CS, Lee I and Chamberlain RS: Trends in incidence, survival, and management of uveal melanoma: A population-based study of 7,516 patients from the Surveillance, Epidemiology, and End Results database (1973-2012). Clin Ophthalmol 10: 2113-2119, 2016.

3. Carvajal RD, Schwartz GK, Tezel T, Marr B, Francis JH and Nathan PD: Metastatic disease from uveal melanoma: Treatment options and future prospects. Br J Ophthalmol 101: 38-44, 2017.

4. Smit KN, Chang J, Derks K, Vaarwater J, Brands T, Verdijk RM, Wiemer EAC, Mensink HW, Pothof J, de Klein A and Kilic E: Aberrant MicroRNA expression and its implications for uveal melanoma metastasis. Cancers (Basel) 11: pii: E815, 2019.

5. Lorenzo D, Piulats JM, Ochoa M, Arias L, Gutiérrez C, Català J, Cobos E, Garcia-Bru P, Dias B, Padrón-Pérez N and Caminal JM: Clinical predictors of survival in metastatic uveal melanoma. Jpn J Ophthalmol 63: 197-209, 2019.

6. Kawaguchi K, Kinameri A, Suzuki S, Senga S, Ke Y and Fujii H The cancer-promoting gene fatty acid-binding protein 5 (FABP5) is epigenetically regulated during human prostate carcinogenesis. Biochem J 473: 449-461, 2016.

7. Ohata T, Yokoo H, Kamiyama T, Fukai M, Aiyama T, Hatanaka Y, Hatanaka K, Wakayama K, Orimo T, Kakisaka T, et al: Fatty acid-binding protein 5 function in hepatocellular carcinoma through induction of epithelial-mesenchymal transition. Cancer Med 6: 1049-1061, 2017.

8. Pan L, Xiao H, Liao R, Chen Q, Peng C, Zhang Y, Mu T and Wu Z: Fatty acid binding protein 5 promotes tumor angiogenesis and activates the IL6/STAT3/VEGFA pathway in hepatocellular carcinoma. Biomed Pharmacother 106: 68-76, 2018.

9. Furuhashi M and Hotamisligil GS: Fatty acid-binding proteins: Role in metabolic diseases and potential as drug targets. Nat Rev Drug Discov 7: 489-503, 2008.

10. Senga S, Kobayashi N, Kawaguchi K, Ando A and Fujii H: Fatty acid-binding protein 5 (FABP5) promotes lipolysis of lipid droplets, de novo fatty acid (FA) synthesis and activation of nuclear factor-kappa $B(N F-\kappa B)$ signaling in cancer cells. Biochim Biophys Acta Mol Cell Biol Lipids 1863: 1057-1067, 2018.

11. Lv Q, Wang G, Zhang Y, Han X, Li H, Le W, Zhang M, Ma C, Wang $P$ and Ding Q: FABP5 regulates the proliferation of clear cell renal cell carcinoma cells via the PI3K/AKT signaling pathway. Int J Oncol 54: 1221-1232, 2019.

12. Wang W, Chu HJ, Liang YC, Huang JM, Shang CL, Tan H, Liu D, Zhao YH, Liu TY and Yao SZ: FABP5 correlates with poor prognosis and promotes tumor cell growth and metastasis in cervical cancer. Tumor Biol 37: 14873-14883, 2016.

13. Armstrong EH, Goswami D, Griffin PR, Noy N and Ortlund EA: Structural basis for ligand regulation of the fatty acidbinding protein 5 , peroxisome proliferator-activated receptor $\beta / \delta$ (FABP5-PPAR $\beta / \delta$ ) signaling pathway. J Biol Chem 289: 14941-14954, 2014.

14. Jeong CY, Hah YS, Cho BI, Lee SM, Joo YT, Jung EJ, Jeong SH Lee YJ, Choi SK, Ha WS, et al: Fatty acid-binding protein 5 promotes cell proliferation and invasion in human intrahepatic cholangiocarcinoma. Oncol Rep 28: 1283-1292, 2012.

15. Kawaguchi K, Senga S, Kubota C, Kawamura Y, Ke Y and Fujii $\mathrm{H}$ : High expression of fatty acid-binding protein 5 promotes cell growth and metastatic potential of colorectal cancer cells. FEBS Open Bio 6: 190-199, 2016

16. Tomczak K, Czerwińska P and Wiznerowicz M: The cancer genome atlas (TCGA): An immeasurable source of knowledge. Contemp Oncol (Pozn) 19: A68-A77, 2015.

17. Camp RL, Dolled-filhart M and Rimm DL: X-tile: A new bio-informatics tool for biomarker assessment and outcome-based cut-point optimization. Clin Cancer Res 10: 7252-7259, 2004.

18. Laurent C, Valet F, Planque N, Silveri L, Maacha S, Anezo O, Hupe P, Plancher C, Reyes C, Albaud B, et al: High PTP4A3 phosphatase expression correlates with metastatic risk in uveal melanoma patients. Cancer Res 71: 666-674, 2011

19. Rhodes DR, Yu J, Shanker K, Deshpande N, Varambally R, Ghosh D, Barrette T, Pandey A and Chinnaiyan AM: ONCOMINE: A cancer microarray database and integrated data-mining platform. Neoplasia 6: 1-6, 2004.

20. Li T, Fan J, Wang B, Traugh N, Chen Q, Liu JS, Li B and Liu XS: TIMER: A web server for comprehensive analysis of tumor-infiltrating immune cells. Cancer Res 77: e108-e110, 2017.

21. Li B, Severson E, Pignon JC, Zhao H, Li T, Novak J, Jiang P, Shen H, Aster JC, Rodig S, et al: Comprehensive analyses of tumor immunity: Implications for cancer immunotherapy. Genome Biol 17: 174, 2016
22. Aran D, Sirota M and Butte AJ: Systematic pan-cancer analysis of tumour purity. Nat Commun 6: 8971, 2015.

23. Franceschini A, Szklarczyk D, Frankild S, Kuhn M, Simonovic M, Roth A, Lin J, Minguez P, Bork P, von Mering C and Jensen LJ: STRING v9.1: Protein-protein interaction networks, with increased coverage and integration. Nucleic Acids Res 41: D808-D815, 2013.

24. Huang DW, Sherman BT, Tan Q, Collins JR, Alvord WG, Roayaei J, Stephens R, Baseler MW, Lane HC and Lempicki RA: The DAVID gene functional classification tool: A novel biological module-centric algorithm to functionally analyze large gene lists. Genome Biol 8: R183, 2007.

25. Subramanian A, Tamayo P, Mootha VK, Mukherjee S, Ebert BL, Gillette MA, Paulovich A, Pomeroy SL, Golub TR, Lander ES and Mesirov JP: Gene set enrichment analysis: A knowledge-based approach for interpreting genome-wide expression profiles. Proc Natl Acad Sci USA 102: 15545-15550, 2005.

26. Dunn GP, Dunn IF and Curry WT: Focus on TILs: Prognostic significance of tumor infiltrating lymphocytes in human glioma. Cancer Immun 7: 12, 2007

27. Azimi F, Scolyer RA, Rumcheva P, Moncrieff M, Murali R, McCarthy SW, Saw RP and Thompson JF: Tumor-infiltrating lymphocyte grade is an independent predictor of sentinel lymph node status and survival in patients with cutaneous melanoma. J Clin Oncol 30: 2678-2683, 2012.

28. Vichitvejpaisal P, Dalvin LA, Mazloumi M, Ewens KG, Ganguly A and Shields CL: Genetic analysis of uveal melanoma in 658 patients using the cancer genome atlas classification of uveal melanoma as A, B, C, and D. Ophthalmology 126: 1445-1453, 2019

29. Caltabiano R, Puzzo L, Barresi V, Ieni A, Loreto C, Musumeci G, Castrogiovanni P, Ragusa M, Foti P, Russo A, et al: ADAM 10 expression in primary uveal melanoma as prognostic factor for risk of metastasis. Pathol Res Pract 212: 980-987, 2016.

30. Broggi G, Musumeci G, Puzzo L, Russo A, Reibaldi M, Ragusa M, Longo A and Caltabiano R: Immunohistochemical expression of ABCB5 as a potential prognostic factor in uveal melanoma. Appl Sci 9: 1316, 2019.

31. Pan J, Dai Q, Zhang T and Li C: Palmitate acid promotes gastric cancer metastasis via FABP5/SP1/UCA1 pathway. Cancer Cell Int 19: 69, 2019.

32. Ju J, Wang $\mathrm{N}$, Wang $\mathrm{J}$, Wu F, Ge J and Chen F: 4-Amino-2-trifluoromethyl-phenyl retinate inhibits proliferation, invasion, and migration of breast cancer cells by independently regulating CRABP2 and FABP5. Drug Des Devel Ther 12 . 997-1008, 2018.

33. Kaczocha M, Vivieca S, Sun J, Glaser ST and Deutsch DG: Fatty acid-binding proteins transport $\mathrm{N}$-acylethanolamines to nuclear receptors and are targets of endocannabinoid transport inhibitors. J Biol Chem 287: 3415-3424, 2012

34. Siegenthaler G, Hotz R, Chatellard-Gruaz D, Didierjean L, Hellman U and Saurat JH: Purification and characterization of the human epidermal fatty acid-binding protein: Localization during epidermal cell differentiation in vivo and in vitro. Biochem J 302: 363-371, 1994

35. Di-Poï N, Michalik L, Tan NS, Desvergne B and Wahli W: The anti-apoptotic role of PPARbeta contributes to efficient skin wound healing. J Steroid Biochem Mol Biol 85: 257-265, 2003.

36. Adhikary T, Brandt DT, Kaddatz K, Stockert J, Naruhn S, Meissner W, Finkernagel F, Obert J, Lieber S, Scharfe M, et al: Inverse PPAR $\beta / \delta$ agonists suppress oncogenic signaling to the ANGPTL4 gene and inhibit cancer cell invasion. Oncogene 32: 5241-5252, 2013

37. Wang D, Wang H, Guo Y, Ning W, Katkuri S, Wahli W, Desvergne B, Dey SK and DuBois RN: Crosstalk between peroxisome proliferator-activated receptor delta and VEGF stimulates cancer progression. Proc Natl Acad Sci USA 103: 19069-19074, 2006.

38. Alshalalfa M, Bismar TA and Alhajj R: Detecting cancer outlier genes with potential rearrangement using gene expression data and biological networks. Adv Bioinformatics 2012: 373506, 2012.

39. Xu Y, Han W, Xu W, Wang Y, Yang XL, Nie HL, Yao J, Shen GL and Zhang XF: Identification of differentially expressed genes and functional annotations associated with metastases ofthe uveal melanoma. J Cell Biochem 120: 19202-19214, 2019.

40. Morgan EA, Forootan SS, Adamson J, Foster CS, Fujii H, Igarashi M, Beesley C, Smith $\mathrm{PH}$ and $\mathrm{Ke} \mathrm{Y}$ : Expression of cutaneous fatty acid-binding protein (C-FABP) in prostate cancer: Potential prognostic marker and target for tumourigenicity-suppression. Int J Oncol 32: 767-775, 2008. 
41. Kowalewska M, Radziszewski J, Goryca K, Bujko M Oczko-Wojciechowska M, Jarzab M, Siedlecki JA and Bidzinski M: Estimation of groin recurrence risk in patients with squamous cell vulvar carcinoma by the assessment of marker gene expression in the lymph nodes. BMC Cancer 12: 223, 2012.

42. Schug TT, Berry DC, Toshkov IA, Cheng L, Nikitin AY and Noy N: Overcoming retinoic acid-resistance of mammary carcinomas by diverting retinoic acid from PPARbeta/delta to RAR. Proc Natl Acad Sci USA 105: 7546-7551, 2008.

43. Levi L, Lobo G, Doud MK, Von Lintig J, Seachrist D, Tochtrop GP and Noy N: Genetic ablation of the fatty acid-binding protein FABP5 suppresses HER2-induced mammary tumorigenesis. Cancer Res 73: 4770-4780, 2013.

44. Andreoli MT, Mieler WF and Leiderman YI: Epidemiological trends in uveal melanoma. Br J Ophthalmol 99: 1550-1553, 2015.
45. Kaliki S, Shields CL and Shields JA: Uveal melanoma: Estimating prognosis. Indian J Ophthalmol 63: 93-102, 2015.

46. Shields CL, Kaliki S, Furuta M, Mashayekhi A and Shields JA Clinical spectrum and prognosis of uveal melanoma based on age at presentation in 8,033 cases. Retina 32: 1363-1372, 2012.

47. Kaliki S, Shields CL, Mashayekhi A, Ganesh A, Furuta M and Shields JA: Influence of age on prognosis of young patients with uveal melanoma: A matched retrospective cohort study. Eur J Ophthalmol 23: 208-216, 2013.

(i) $($ ) This work is licensed under a Creative Commons Attribution-NonCommercial-NoDerivatives 4.0 International (CC BY-NC-ND 4.0) License. 